\title{
On the structure of (pan, even hole)-free graphs*
}

\author{
Kathie Cameron ${ }^{1}$ \\ Steven Chaplick ${ }^{2 \dagger}$ \\ Chính T. Hoàng ${ }^{3}$
}

\author{
${ }^{1}$ Department of Mathematics, Wilfrid Laurier University, Waterloo, Ontario, Canada, N2L 3C5 \\ kcameron@wlu.ca \\ ${ }^{2}$ Lehrstuhl für Informatik I Universität Würzburg, Am Hubland D-97074 Würzburg, Germany \\ steven. chaplick@uni-wuerzburg.de \\ ${ }^{3}$ Department of Physics and Computer Science, Wilfrid Laurier University, Waterloo, Ontario, Canada, \\ N2L 3C5 \\ choang@wlu.ca
}

\begin{abstract}
A hole is a chordless cycle with at least four vertices. A pan is a graph which consists of a hole and a single vertex with precisely one neighbor on the hole. An even hole is a hole with an even number of vertices. We prove that a (pan, even hole)-free graph can be decomposed by clique cutsets into essentially unit circular-arc graphs. This structure theorem is the basis of our $O(n m)$-time certifying algorithm for recognizing (pan, even hole)-free graphs and for our $O\left(n^{2.5}+n m\right)$-time algorithm to optimally color them. Using this structure theorem, we show that the tree-width of a (pan, even hole)-free graph is at most 1.5 times the clique number minus 1 , and thus the chromatic number is at most 1.5 times the clique number.
\end{abstract}

\section{Introduction}

A hole is a chordless cycle with at least four vertices. A graph is chordal if it does not contain a hole as an induced subgraph. Chordal graphs are well-studied and have a number of interesting structural properties (see [3, 18]). For example, it is known [14] that every chordal graph contains a simplicial vertex; i.e., a vertex whose neighborhood induces a clique. Based on this, a largest clique, a minimum coloring, a largest stable set, and a minimum partition into cliques of a chordal graph can be found in polynomial time [17.

An even hole is a hole with an even number of vertices. A graph is even-hole-free if it does not contain an even hole as an induced subgraph. Even-hole-free graphs generalize chordal graphs and analogous properties have been found. A largest clique of an even-holefree graph can be found in polynomial time [1, 2, 13. However, it is not known whether even-hole-free graphs can be optimally colored in polynomial time.

The claw is the graph with vertices $a, b, c, d$ and edges $a b, a c, a d$. As usual, $n$ (respectively, $m$ ) denotes the number of vertices (respectively, edges) of the input graph $G$.

\footnotetext{
${ }^{*}$ Research support by Natural Sciences and Engineering Research Council of Canada.

${ }^{\dagger}$ Research partially supported by the European Science Foundation project EUROGIGA GraDR.
} 
We give an $O\left(n^{2.5}+n m\right)$-time algorithm to color (claw, even hole)-free graphs, providing a contrast to the well-known result [19] that it is NP-hard to optimally color claw-free graphs. Our techniques actually apply to a larger class of graphs which we will now define. An atom is a connected graph without a clique cutset. A pan is a graph which consists of a hole and a single vertex with precisely one neighbor on the hole. Let $\mathcal{C}$ denote the class of graphs $G$ such that each atom of $G$ is (pan, even hole)-free. In this paper, we will give an $O\left(n^{2.5}+n m\right)$-time algorithm to color a graph in $\mathcal{C}$, and an $O(n m)$-time certifying algorithm for recognition of graphs in $\mathcal{C}$ and recognition of (pan, even hole)-free graphs. Pan-free graphs have been studied previously regarding: establishing the perfectness of (pan, odd hole)-free graphs [27], and providing a polynomial-time algorithm to find a largest weight stable set first on a subclass of pan-free graphs [15] and then the whole class of pan-free graphs [4. The latter two papers use the term "apple" for "pan".

In Section 2, we will cover the relevant background and state our main results. In Section 3, we will prove that a (pan, even hole)-free graph can be decomposed by the well-studied clique cutset decomposition into, essentially, "unit circular-arc graphs". This structural result is the foundation of our polynomial-time algorithms. In Section 4, we give our $O\left(n^{2.5}+n m\right)$-time algorithm for coloring the graphs in $\mathcal{C}$. In Section 5 , we discuss our $O(n m)$-time algorithm to recognize if a graph is in $\mathcal{C}$. In Section 6, we show that the tree-width of a (pan, even hole)-free graph is at most 1.5 times the clique number minus 1 , and thus the chromatic number is at most 1.5 time the clique number. In Section 7, we discuss open problems related to our work.

\section{Background and results}

In this section, we discuss the relevant background and give the definitions necessary to state our main results. Let $G$ be a graph. For a subset $S$ of the vertices of $G$, we use $G[S]$ to denote the subgraph of $G$ induced by $S$. A clique cutset of $G$ is a set $S$ of vertices where $G[S]$ is a clique whose removal increases the number of components of $G$. The following theorem is well known.

Theorem 2.1 [14 Every chordal graph is either a clique or contains a clique cutset.

Recall that a vertex is simplicial if its neighborhood induces a clique, and a vertex is bi-simplicial if its neighborhood can be partitioned into two cliques (i.e., its neighborhood induces the complement of a bipartite graph). It follows from Theorem 2.1 that every chordal graph contains a simplicial vertex.

Let $d_{G}(x)$ denote the degree of a vertex $x$ in a graph $G$. When the context is clear, we will write $d(x)$ to mean $d_{G}(x)$. Let $\chi(G)$ (respectively, $\omega(G)$ ) denote the chromatic number (respectively, the clique number, i.e., the number of vertices in a largest clique) of $G$. If $v$ is a simplicial vertex of $G$, then $\chi(G)=\max (\chi(G-v), d(v)+1)$ and $\omega(G)=$ $\max (\omega(G-v), d(v)+1)$. An analogous property was established for even-hole-free graphs. (Recall a hole is even (odd) if it has an even (odd) number of vertices.)

Theorem 2.2 [2] Every even-hole-free graph contains a bi-simplicial vertex.

Theorem 2.2 implies that for even-hole-free graphs $G$, a largest clique can be found in polynomial time and that $\chi(G) \leq 2 \omega(G)-1$. However, it is not known if the coloring problem can be solved in polynomial time for even-hole-free graphs.

The clique (respectively, chordless cycle, chordless path) on $t$ vertices is denoted by $K_{t}$ (respectively, $C_{t}, P_{t}$ ). Recall that the claw is the graph with vertices $a, b, c, d$ and edges $a b, a c, a d$; vertex $a$ is the center of the claw. Let $F$ be a graph and let $\mathcal{F}$ be a family 
of graphs. We say that a graph $G$ is $F$-free if $G$ does not contain an induced subgraph isomorphic to $F$ and $G$ is $\mathcal{F}$-free if $G$ does not contain an induced subgraph isomorphic to any graph in $\mathcal{F}$. In particular, $G$ is (claw, even hole)-free if $G$ does not contain a claw or an even hole as an induced subgraph.

Theorem 2.2 implies that an even-hole-free graph contains a vertex that is not the center of a claw. This suggests that even-hole-free graphs such that no vertex is the center of a claw (i.e., (claw, even hole)-free graphs) might have interesting structure. Indeed, our results show that (claw, even hole)-free graphs can be decomposed by the clique cutset decomposition into (essentially) unit circular-arc graphs. Our results actually apply to a larger class of graphs that we will define later in this section (see Theorem 2.3).

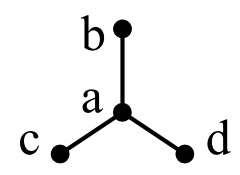

Figure 1: The claw with center $a$.

Consider the following procedure to decompose a graph $G$. If $G$ has a clique cutset $C$, then $G$ can be decomposed into subgraphs $G_{1}=G\left[V_{1}\right]$ and $G_{2}=G\left[V_{2}\right]$ where $V=V_{1} \cup V_{2}$ and $C=V_{1} \cap V_{2}$. Note: there is no edge between $\left(G_{1}-C\right)$ and $\left(G_{2}-C\right)$. Given minimum colorings of $G_{1}$ and $G_{2}$, we can obtain a minimum coloring of $G$ by identifying the coloring of $C$ in $G_{1}$ with that of $C$ in $G_{2}$. In particular, we have $\chi(G)=\max \left(\chi\left(G_{1}\right), \chi\left(G_{2}\right)\right)$. If $G_{i}$ $(i \in\{1,2\})$ has a clique cutset, then we can recursively decompose $G_{i}$ in the same way. This decomposition can be represented by a binary tree $T(G)$ whose root is $G$ and where the two children of $G$ are $G_{1}$ and $G_{2}$, which are in turn the roots of subtrees representing the decompositions of $G_{1}$ and $G_{2}$. Each leaf of $T(G)$ corresponds to an induced subgraph of $\mathrm{G}$ that contains no clique cutset; such an induced graph is called an atom of $G$. Algorithmic aspects of the clique cutset decomposition are studied in 33 and 35. In particular, the decomposition tree $T(G)$ can be constructed in $O(n m)$ time such that the total number atoms is at most $n-1$ [33]. We have seen in the discussion above that the clique cutset decomposition can be used to color a graph. With $G, G_{1}$, and $G_{2}$ defined as above, $G$ contains an even hole (or odd hole) if and only if $G_{1}$ or $G_{2}$ does. Thus, the clique cutset decomposition can also be used to find an even hole (or odd hole), if one exists.

Recall that a pan is the graph obtained from taking a $C_{k}$ with $k \geq 4$, adding another vertex $x$, and joining $x$ to a vertex $y$ of the $C_{k}$ by an edge. The edge $x y$ is called the handle of the pan. We will show that (pan, even hole)-free atoms have very special structure. This structure allows us to solve the recognition and coloring problems. To describe this structure, we will need to introduce more definitions.

A graph $G$ is a circular-arc graph if there is a bijection between its vertices and a set $A$ of arcs on a circle such that two vertices of $G$ are adjacent if and only if the two corresponding arcs of $A$ intersect. A circular-arc graph is proper if no arc contains another. Additionally, $G$ is a unit circular-arc graph if every arc of $A$ has the same length. It is easy to see that unit circular-arc graphs are proper and that proper circular-arc graphs are claw-free and hence pan-free.

Let $A$ and $B$ be two disjoint sets of vertices. We say $A$ is $B$-null if there is no edge between $A$ and $B$, and $A$ is $B$-complete if every possible edge between $A$ and $B$ is present. For a vertex $x, N_{G}(x)$ denotes the set of neighbors of $x$ in $G$. When the context is obvious, we use $N(x)$ for $N_{G}(x)$. For a set $X$ of vertices, $N(X)$ denotes the set of vertices outside $X$ that have neighbors in $X$. A vertex $a$ dominates a vertex $b$ if $(N(b)-\{a\}) \subseteq N(a)$. Vertex a strictly dominates vertex $b$ if $(N(b)-\{a\}) \subsetneq N(a)$. Two vertices are comparable 
if one dominates the other. The domination relation is transitive, that is, if $a$ dominates $b$ and $b$ dominates $c$, then $a$ dominates $c$. Thus, given a set $X$ of vertices such that any two vertices in $X$ are comparable, there is a total order $\prec$ on $X$ such that $a \prec b$ whenever $a$ dominates $b$. We call such order a domination order. Two vertices $a$ and $b$ are comparable in $X$ if they are comparable in the subgraph induced by $X \cup\{a, b\}$.

Let $A$ and $B$ be two vertex-disjoint graphs. The join of $A$ and $B$ is the graph $C$ obtained from $A$ and $B$ by adding every edge between the vertices of $A$ and those of $B$; thus, in $C$ the vertices of $A$ are $B$-complete and vice versa.

We now state our decomposition theorem for the class $\mathcal{C}$.

Theorem 2.3 If $G$ is a connected graph in $\mathcal{C}$ (i.e. every atom of $G$ is (pan, even hole)free), then

(i) $G$ is a clique, or

(ii) $G$ contains a clique cutset, or

(iii) $G$ is a unit circular-arc graph, or

(iv) $G$ is the join of a unit circular-arc graph and a clique.

In [6], a polynomial-time algorithm is given for finding an even hole (or, odd hole) in a circular-arc graph. In [28] or combining [24] and 31], polynomial-time algorithms are given for finding an optimal coloring of a unit circular-arc graph. In Sections 4 and 5 , we discuss how these results can be used to color and recognize both the class $\mathcal{C}$ and the class of (pan, even hole)-free graphs. In Section 3, we will establish structural properties of (pan, even hole)-free graphs. Specifically, we will prove Theorem 2.3.

Our main result is:

Theorem 2.4 Given a graph $G$, a pan or even hole of $G$, if one exists, can be found in $O(n m)$ time.

We end this section with a discussion on the relationship between even-hole-free graphs and $\beta$-perfect graphs, which were introduced in [26] and are defined as follows. Let $\delta(G)$ denote the minimum degree of a vertex of a graph $G$. Order the vertices of $G$ as $v_{1}, \ldots, v_{n}$ where $v_{i}$ has minimum degree in $G\left[v_{i}, \ldots, v_{n}\right]$. Greedily color $G$ starting from $v_{n}$, i.e., $v_{i}$ is given the smallest color distinct from its neighborhood in $G\left[v_{i}, \ldots, v_{n}\right]$. The number of colors used is at most maximum $\{\delta(H)+1: H$ is an induced subgraph of $G\}$, which is denoted $\beta(G)$. Thus for any graph $G$, we have $\chi(G) \leq \beta(G)$. A graph is defined to be $\beta$-perfect, if for every induced subgraph $F$ of $G, \chi(F)=\beta(F)$. An even hole $C_{2 k}$ has $\chi\left(C_{2 k}\right)=2$ and $\beta\left(C_{2 k}\right)=3$. It follows that $\beta$-perfect graphs are even-hole-free. A diamond is the complete graph on four vertices with an edge removed. In [21], it is proved that (diamond, even hole)-free graphs are $\beta$-perfect. In [26], the authors gave the graph of Figure 2 as an example of an even-hole-free graph which is not $\beta$-perfect. This graph is claw-free and hence pan-free, so it follows that (claw, even hole)-free and thus (pan, even hole)-free graphs need not be $\beta$-perfect.

\section{Properties of (pan, even hole)-free graphs}

In this section we will prove our structure theorem (Theorem 2.3). We will actually prove a stronger but more technical result which implies Theorem 2.3 (see Theorem 3.17). We separate the discussion into two subsections. In the first we consider a special substructure of a graph which generalizes holes: we call this substructure a "buoy". In the second we prove that (pan, even hole)-free graphs decompose into buoys via clique cutsets. 


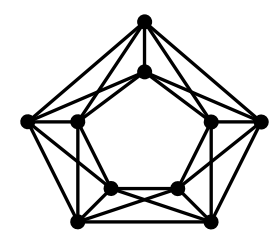

Figure 2: A claw-free, non- $\beta$-perfect graph

\subsection{Buoys}

To motivate our buoys we start with a key observation regarding the structure around a hole in a pan-free graph.

Observation 3.1 Let $G$ be a pan-free graph. Let $C$ be a hole of $G$ of length at least five and let $x$ be a vertex outside $C$.

(i) If $x$ has a neighbor $v$ in $C$, then some $u \in C$ is adjacent to both $v$ and $x$.

(ii) If $x$ has exactly three neighbors $v_{1}, v_{2}, v_{3}$ in $C$, then $v_{1}, v_{2}, v_{3}$ forms a path in $C$.

(iii) If $x$ has exactly four neighbors in $C$, then $G$ contains an even hole.

(iv) If $x$ has at least five neighbors in $C$, then $x$ is $C$-complete.

(v) If $G$ is even-hole-free, then $x$ has 2, 3, or $\ell$ neighbors in $C$, where $\ell$ is the length of $C$.

Proof: Enumerate the vertices of $C$ in the cyclic order as $v_{0}, v_{1}, \ldots, v_{\ell-1}$. Suppose that (i) is false for a neighbor $v$ of $x$. We may assume $v=v_{0}$, i.e., $x$ is adjacent to $v_{0}$, and non-adjacent to $v_{1}$ and $v_{\ell-1}$. Vertex $x$ must have another neighbor in $C$, for otherwise $x$ and $C$ form a pan. Let $k$ be the smallest subscript, different from 0 , such that $v_{k}$ is a neighbor of $x$. If $k=\ell-2$, then $\left\{v_{1}, v_{0}, v_{\ell-1}, v_{\ell-2}, x\right\}$ induces a pan. If $k \neq \ell-2$, then $\left\{v_{\ell-1}, v_{0}, v_{1}, \ldots, v_{k}, x\right\}$ induces an pan. We have established (i). Now (ii) follows immediately from (i). For (iii) suppose $C$ is an odd hole (otherwise, we are done). Now, by (i), these four vertices either form single sub-path $v_{i} v_{i+1} v_{i+2} v_{i+3}$ of $C$ or two non-adjacent paths $v_{i} v_{i+1}$ and $v_{t} v_{t+1}$ such that $C$ can be written as $v_{i+1} P_{1} v_{t} v_{t+1} P_{2} v_{i}$ (for non-empty paths $P_{1}, P_{2}$ ). In the former case, an even hole is induced by $\{x\} \cup C-\left\{v_{i+1} v_{i+2}\right\}$. In the latter case, one of $x v_{i+1} P_{1} v_{t}$ and $x v_{t+1} P_{2} v_{i}$ is an even hole. We now prove (iv). Suppose $x$ has at least five neighbors in $C$ but is not $C$-complete (this implies $\ell>5$ ). We may assume $x$ is adjacent to $v_{0}$ and non-adjacent to $v_{1}$. Let $k$ be the smallest subscript, different from 0 , such that $v_{k}$ is a neighbor of $x$. By (i), $x$ is adjacent to $v_{k+1}$ and $v_{\ell-1}$. Since $x$ has at least five neighbors in $C, x$ is adjacent to a vertex $v_{t}$ with $k+1<t<\ell-1$. But now $\left\{v_{t}, x, v_{0}, v_{1}, \ldots, v_{k}\right\}$ induces a pan. Part (v) follows from (i)-(iv).

For the purpose of finding a forbidden induced subgraph for recognition of class $\mathcal{C}$, we will now reformulate the results of Observation 3.1 into their algorithmic counter-parts. From the proof of Observation 3.1, we can extract a linear-time algorithm to find a pan or even hole of an input graph when one of the conditions (i)-(v) fails.

Observation 3.2 Let $G$ be a graph, let $C$ be a hole of $G$ of length at least five, and let $x$ be a vertex outside $C$. If $x$ fails to satisfy (i)-(v) of Observation 3.1, then $G$ contains a pan or an even hole, and such an induced graph can be found in linear time. 
We generalize the presence of a length $\ell \geq 5$ hole (and Observation 3.1) in a graph to the presence of an $\ell$-buoy (defined as follows). For $\ell \geq 5$, an $\ell$-buoy $B$ is a collection of sets $B_{0}, B_{1}, \ldots, B_{\ell-1}$ of vertices of $G$ such that each $B_{i}$ induces a clique, each vertex in $B_{i}$ has a neighbor in $B_{i+1}$ and one in $B_{i-1}$, and there are no edges between $B_{i}$ and $B-\left(B_{i-1} \cup B_{i} \cup B_{i+1}\right)$, with subscripts taken modulo $\ell$ (see Figure 3 for an example); the sets $B_{i}$ are called the bags of the buoy; a buoy is odd or even depending on whether the number of bags $(\ell)$ is odd or even. We also refer to $G[B]$ as a buoy. A skeleton of $B$ is a hole containing one vertex of each $B_{j}, j \in\{0, \ldots, \ell-1\}$. A buoy $B$ in a graph $G$ is said to be full when it includes every vertex of $G$. Due to the cyclic structure of $\ell$-buoys, when we refer to a bag $B_{i}$ of an $\ell$-buoy, we always mean the bag $B_{i}(\bmod \ell)$. We will see that when $G$ is $C_{4}$-free its buoys are circular-arc graphs (see Theorem 3.8). Similarly, when $G$ is (pan, even hole)-free its buoys are unit circular-arc graphs (see Theorem 3.14).

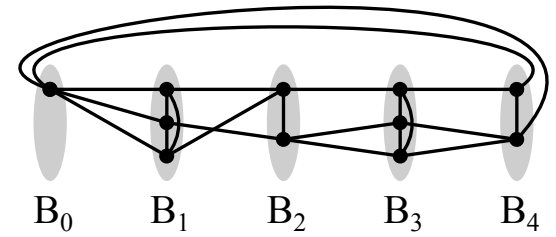

Figure 3: An example of a 5-buoy.

Observation 3.3 Let $G$ be an even-hole-free graph having an odd $\ell$-buoy with bags $B_{0}, \ldots, B_{\ell-1}$. Consider a path $b_{0}, b_{1}, \ldots, b_{k-1}$ with $1 \leq k \leq \ell$ where $b_{i} \in B_{i}$ for $i=0, \ldots, k-1$. Then where $z=\min \{k-1, \ell-2\},\left\{b_{0}, \ldots, b_{z}\right\}$ belongs to a skeleton of $B$.

Proof: By definition of the buoy, there is an induced path $b_{k-1}, b_{k}, \ldots, b_{\ell-1}$ such that $b_{j} \in B_{j}$ for $j=k, k+1, \ldots, \ell-1$. We may assume $b_{0}$ is not adjacent to $b_{\ell-1}$, for otherwise we are done. Let $y$ be a neighbor of $b_{0}$ in $B_{\ell-1}$. We have $b_{\ell-2} y \in E(G)$, for otherwise $\left\{b_{0}, b_{1} \ldots, b_{\ell-2}, b_{\ell-1}, y\right\}$ induced an even hole. If $k=\ell$, then $\left\{b_{0}, \ldots, b_{k-2}, y\right\}$ induces a skeleton; if $k<\ell$, then $\left\{b_{0}, \ldots, b_{k-2}, b_{k-1}, \ldots, b_{\ell-2}, y\right\}$ induces a skeleton.

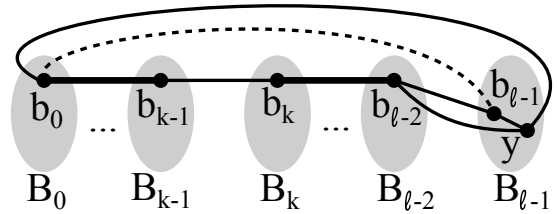

Figure 4: The skeleton containing the path $b_{0}, \ldots, b_{z}$ where $z=\min \{k-1, \ell-2\}$ as in the proof of Observation 3.3 . Note: the bold edges connecting $b_{0}$ to $b_{k-1}$ and $b_{k}$ to $b_{\ell-2}$ correspond to the paths connecting these vertices.

Corollary 3.4 Let $G$ be an even-hole-free graph having an odd $\ell$-buoy with bags $B_{0}, \ldots$, $B_{\ell-1}$. Let $P$ be a path with vertices $p_{i}, p_{i+1}, \ldots, p_{i+k}$ where $0 \leq k \leq \ell-2$ and $p_{j} \in B_{j}$ for all $j$ (with the subscripts taken modulo $\ell$ ), then $P$ belongs to a skeleton of $B$.

From the proof of Observation 3.3, we can extract a linear-time algorithm to establish the following observation.

Observation 3.5 Let $G$ be a graph having an odd $\ell$-buoy with bags $B_{0}, \ldots, B_{\ell-1}$. Consider a path $b_{0}, b_{1}, \ldots, b_{k-1}$ with $1 \leq k \leq \ell$ where $b_{i} \in B_{i}$ for $i=0, \ldots, k-1$. Then there is a linear-time algorithm that either finds an even hole, or a skeleton containing the set $\left\{b_{0}, \ldots, b_{z}\right\}$ (for a given $z=\min \{k-1, \ell-2\}$ ). 
Observation 3.6 Let $G$ be a $C_{4}$-free graph. Let $B$ be an $\ell$-buoy of $G$ with bags $B_{0}, \ldots, B_{\ell-1}$. Then any two vertices $a$ and $b$ in the same $B_{i}$ are comparable in $B_{i-1}$. By symmetry, $a$ and $b$ are comparable in $B_{i+1}$.

Proof: Let $a$ and $b$ be two vertices in $B_{i}$. Suppose they are not comparable in $B_{i-1}$. Then there are vertices $x, y \in B_{i-1}$ with $x a, y b \in E(G)$ and $x b, y a \notin E(G)$. Now, the four vertices $a, b, x, y$ form a $C_{4}$.

From the proof of Observation 3.6, we can extract a linear-time algorithm to establish the following observation.

Observation 3.7 Let $G$ be a graph. Let $B$ be an $\ell$-buoy of $G$ with bags $B_{0}, \ldots, B_{\ell-1}$. If two vertices $a$ and $b$ in the same $B_{i}$ are not comparable in $B_{i-1}$, then $G$ contains a $C_{4}$, and this $C_{4}$ can be found in linear time.

Theorem 3.8 If $B$ is an $\ell$-buoy of a $C_{4}$-free graph $G$, then $B$ is a circular-arc graph.

Proof: Let $B_{0}, \ldots, B_{\ell-1}$ be the bags of $B$. We construct a circular-arc representation of $G$ as follows. First we partition the circle into $\ell$ arcs of equal length and label the boundary points of these arcs as $(0),(1), \ldots,(\ell-1)$ in clockwise order. By Observation 3.6 the vertices of $B_{i}$ can be partitioned and ordered by neighborhood inclusion with respect to $B_{i+1}$.

That is, we let $B_{i}=\bigcup_{j=1}^{t_{i}} B_{i, j}$ such that for $v, v^{\prime} \in B_{i, j}, N(v) \cap B_{i+1}=N\left(v^{\prime}\right) \cap B_{i+1}$ and for $u \in B_{i, j}$ and $x \in B_{i, j+1}$, the neighborhood of $u$ is a strict subset of that of $x$ with respect to $B_{i+1}$ (i.e., $N(u) \cap B_{i+1} \subsetneq N(x) \cap B_{i+1}$ ). That is, $B_{i}$ can be partitioned into $t_{i}$ subsets $B_{i, 1}, \ldots, B_{i, t_{i}}$ where: (1) if $v, v^{\prime} \in B_{i, j}$, then they have the same neighbors in $B_{i+1}$ (i.e., $N(v) \cap B_{i+1}=N\left(v^{\prime}\right) \cap B_{i+1}$ ), and (2) if $u \in B_{i, j}$ and $v \in B_{i, j+1}$, then in $B_{i+1}$ the neighborhood of $u$ is a strict subset of the of the neighborhood of $v$ (i.e., $\left.N(u) \cap B_{i+1} \subsetneq N(v) \cap B_{i+1}\right)$. From this partitioning of $B_{i}$ and $B_{i+1}$ we can easily construct arcs between $(i)$ and $(i+1)$ to capture the edges between vertices of $B_{i}$ and $B_{i+1}$. This is depicted in Figure 5 and described as follows.

For every $i$, we place $t_{i}$ equally spaced points $\left\{(i, 1), \ldots,\left(i, t_{i}\right)\right\}$ on the arc from $(i)$ to $(i+1)$ and:

- for a vertex $b_{j}$ in $B_{i, j}$ we use the arc from $(i)$ to $(i, j)$.

- for a vertex $x_{j}$ in $X_{i+1, j}$ we use the arc from $(i, j)$ to $(i+1)$.

Clearly, the arc from $(i)$ to $(i, j)$ precisely intersects all arcs from vertices in $B_{i}$ and the arcs of $b_{j}$ 's neighbors in $B_{i+1}$. Similarly, the arc from $(i, j)$ to $(i+1)$ precisely intersects all arcs from vertices in $B_{i+1}$ and the arcs of $x_{j}$ 's neighbors in $B_{i}$.

Notice that, for each $v \in B$ there is a unique triple $\left(i, j, j^{\prime}\right)$ of indices where $v \in$ $B_{i, j} \cap X_{i, j^{\prime}}$. Thus, by performing this construction for each $i \in\{0, \ldots, \ell-1\}$, each $v$ will be mapped to an arc from $\left(i-1, j^{\prime}\right)$ to $(i, j)$; i.e., we have a circular-arc representation for $B$.

Let $B$ be an $\ell$-buoy of a graph $G$ with bags $B_{0}, \ldots, B_{\ell-1}$. Consider a vertex $x$ of some bag $B_{i}$. We say $x$ is a dominant vertex of $B_{i}$ if (in $B$ ) it dominates every other vertex of $B_{i}$.

Observation 3.9 Let $B$ be an odd $\ell$-buoy of an even-hole-free graph $G$ with bags $B_{0}, \ldots, B_{\ell-1}$. For every $i \in\{0, \ldots, \ell-1\}$ and every pair of vertices $x, y$ in $B_{i}, x$ and $y$ are comparable in $B$. In particular, each $B_{i}$ contains a dominant vertex. 


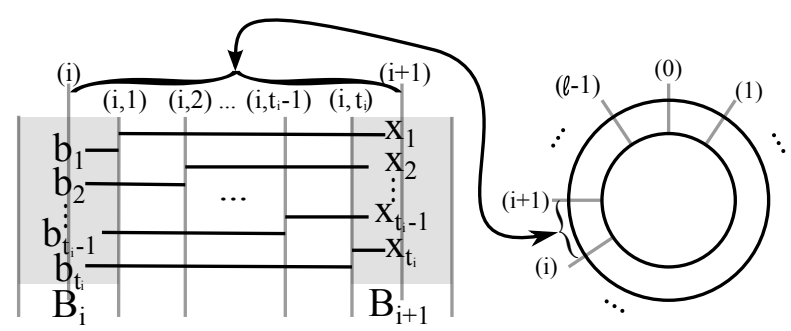

Figure 5: The partial arcs between $(i)$ and $(i+1)$ where the $\operatorname{arc} b_{j}$ represents the vertices from $B_{i, j}$ and the arc $x_{j}$ represents the vertices from $X_{i+1, j}$.

Proof: Figure 6 depicts the structure we observe in this proof. Suppose some pair of vertices $x, y$ in $B_{0}$ are incomparable. Then by Observation 3.6, there are vertices $a \in$ $B_{1}, b \in B_{\ell-1}$ with $x a, b y \in E(G)$ and $y a, b x \notin E(G)$. Now, by Observation 3.3 and for the edges $x a$ and $b y, B$ has skeletons $\left(x, a\left(=a_{1}\right), a_{2}, \ldots, a_{\ell-1}\right)$ and $\left(y, b\left(=b_{\ell-1}\right), \ldots, b_{1}\right)$ where $a_{i}, b_{i} \in B_{i}$.

Notice that if $a_{i} b_{i+1}$ is an edge, then $\left(y, x, a_{1}, a_{2}, a_{3}, \ldots, a_{i}, b_{i+1}, \ldots, b_{\ell-1}\right)$ is an even hole. Thus, $a_{i} b_{i+1} \notin E(G)$ and each $a_{i}$ is distinct from each $b_{j}$. Moreover, $b_{i} a_{i+1}$ is an edge (otherwise $a_{i}, b_{i}, b_{i+1}, a_{i+1}$ is an induced $C_{4}$ ). But now $\left(x, a_{1}, a_{2}, b_{2}, b_{3}, \ldots, b_{\ell-2}, a_{\ell-1}\right)$ is an even hole since $a_{1} b_{2}, a_{2} b_{3} \notin E(G), a_{2} \neq b_{2}$, and $b_{\ell-2} a_{\ell-1} \in E(G)$, a contradiction. Since the domination relation is transitive, every bag $B_{i}$ contains a vertex $d_{i}$ that dominates every other vertex of $B_{i}$, i.e., $d_{i}$ is a dominant vertex of $B_{i}$

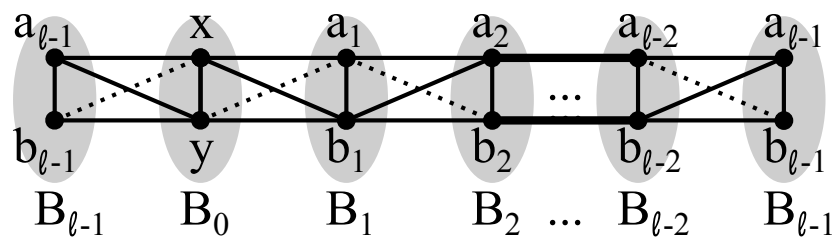

Figure 6: The $\ell$-buoy from the proof of Observation 3.9. Note: we have duplicated bag $B_{\ell-1}$ for ease of presentation. Also, the bold edges connecting $a_{2}$ with $a_{\ell-2}$ and $b_{2}$ with $b_{\ell-2}$ correspond the to the paths $a_{2}, \ldots, a_{\ell-2}$ and $b_{2}, \ldots, b_{\ell-2}$ respectively.

From the proof of Observation 3.9, we can extract a linear-time algorithm to establish the following observation.

Observation 3.10 Let $B$ be an odd $\ell$-buoy of a graph $G$ with bags $B_{0}, \ldots, B_{\ell-1}$. If there are vertices $x$ and $y$ in some $B_{i}$ such that $x$ and $y$ are incomparable in $B$, then $G$ contains an even hole, and this even hole can be found in linear time.

Later (Lemma 5.7) we will show that the domination property of Observation 3.9 can be verified in linear time.

Observation 3.11 Let $G$ be a (pan, even hole)-free graph. Let $B$ be an $\ell$-buoy of $G$ with bags $B_{0}, \ldots, B_{\ell-1}$. Let $a$ and $b$ be two vertices in some $B_{i}$. If a strictly dominates $b$ in $B_{i+1}$, then $b$ dominates a in $B_{i-1}$.

Proof: Let $a$ and $b$ be two vertices in $B_{i}$. Suppose $a$ strictly dominates $b$ in $B_{i+1}$, but $b$ does not dominate $a$ in $B_{i-1}$. Thus, there are vertices $c \in B_{i+1}, d \in B_{i-1}$ such that $a c, a d \in E(G)$ and $b c, b d \notin E(G)$. By Corollary 3.4, there is a skeleton $C$ containing the vertices $d, a, c$. Now $b$ together with $C$ induces a pan in $G$. 
From the proof of Observation 3.11, we can extract an algorithm to establish the following observation.

Observation 3.12 Let $G$ be a graph. Let $B$ be an $\ell$-buoy of $G$ with bags $B_{0}, \ldots, B_{\ell-1}$. Let $a$ and $b$ be two vertices in some $B_{i}$. If a strictly dominates $b$ in $B_{i+1}$, and $b$ does not dominate $a$ in $B_{i-1}$, then $G$ contains a pan or an even hole, and such an induced subgraph can be found in linear time.

Observations 3.11 and 3.9 tell us that the structure of a buoy in a (pan, even hole)-free graph is very restricted (see Corollary 3.13 below). Additionally, this structure allows us to prove that a buoy in a (pan, even hole)-free graph is a unit circular-arc graph (see Theorem 3.14 below).

Corollary 3.13 Let $G$ be a (pan, even hole)-free graph and let $B$ be an $\ell$-buoy of $G$ with bags $B_{0}, \ldots, B_{\ell-1}$. For every $i \in\{0, \ldots, \ell-1\}$ either $B_{i-1} \cup B_{i}$ or $B_{i} \cup B_{i+1}$ is a clique.

Proof: Consider a bag $B_{i}$. By Observation 3.9, we can order the vertices of $B_{i}$ as $b_{i, 1}, b_{i, 2}, \ldots, b_{i, t}$ such that $b_{i, k}$ dominates $b_{i, j}$ whenever $k>j$. In particular, the vertex $b_{i, t}$ is a dominant vertex of $B_{i}$, and $b_{i, t}$ is adjacent to all of $B_{i-1} \cup B_{i+1}$. We may suppose $B_{i} \cup B_{i+1}$ is not a clique, for otherwise we are done. Consider a vertex $b_{i, r}$ that is not adjacent to some vertex $u$ in $B_{i+1}$. Thus, $b_{i, t}$ strictly dominates $b_{i, r}$, and therefore $b_{i, 1}$. By Observation 3.11, $b_{i, 1}$ dominates $b_{i, t}$ in $B_{i-1}$, and so $b_{i, 1}$ is adjacent to all of $B_{i-1}$. By the domination order of $B_{i}$, every other vertex in $B_{i}$ is adjacent to all of $B_{i-1}$. Thus $B_{i-1} \cup B_{i}$ induces a clique.

Theorem 3.14 If $B$ is an $\ell$-buoy in a (pan, even hole)-free graph $G$, then $B$ is a unit circular-arc graph.

Proof: This proof is an easy adaptation of the construction from the proof of Theorem 3.8 . By Corollary 3.13, when $B_{i} \cup B_{i+1}$ is not a clique, both $B_{i-1} \cup B_{i}$ and $B_{i+1} \cup B_{i+2}$ must be cliques. In particular, when $B_{i} \cup B_{i+1}$ is not a clique we use nearly the same construction as before and exploit the fact that $B_{i-1} \cup B_{i}$ and $B_{i+1} \cup B_{i+2}$ are cliques to ensure all of our arcs have the same length. Figure 7 depicts our construction and we will refer to it as we describe the details.

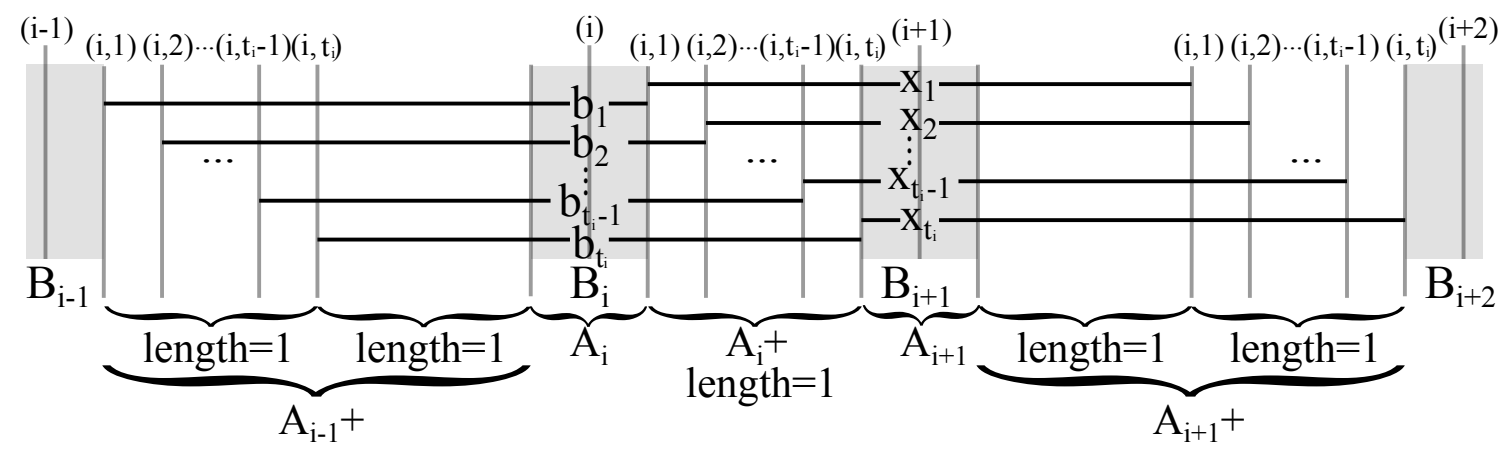

Figure 7: The unit circular-arc construction from the proof of Theorem 3.14 for the case when $B_{i} \cup B_{i+1}$ is not a clique.

The arcs we construct will have length $2+\epsilon$. As in the previous case we first partition the circle into arcs. We then use these arcs to place the endpoints of the arcs for the vertices of $B$. 
We partition the circle into arcs as follows. For each bag $B_{i}$ we allocate an arc $A_{i}$ of length $\epsilon$. The midpoint of $A_{i}$ will be the point $(i)$ from the proof of Theorem 3.8. For each $i \in\{0, \ldots, \ell-1\}$ we allocate an arc $A_{i}+$ such that:

- When $B_{i} \cup B_{i+1}$ is a clique, the length of $A_{i}+$ is two.

- When $B_{i} \cup B_{i+1}$ is not a clique, the length of $A_{i}+$ is one.

These arcs are arranged as $A_{0}, A_{0}+, A_{1}, A_{1}+, \ldots, A_{\ell-1}, A_{\ell-1}+$ around the circle so that the circle is covered and consecutive arcs intersect in precisely one point.

As we mentioned, when $B_{i} \cup B_{i+1}$ is not a clique we use the previous construction subject to the constraint that the length of the arc from $(i, 1)$ to $\left(i, t_{i}\right)$ is one (note: $t_{i}>1$ since $B_{i} \cup B_{i+1}$ is not a clique).

In the first half of $A_{i-1}+$ we insert a copy of the points of $A_{i}+$. In particular, the left endpoint of $A_{i-1}+$ is a copy of $(i, 1)$ and this is followed by $(i, 2), \ldots,\left(i, t_{i}-1\right),\left(i, t_{i}\right)$ with precisely the same spacing as in $A_{i}+$. With these points we can now create the arcs for the vertices in $B_{i}$. Specifically, using the same partition $B_{i, j}$ as before, each $b_{j} \in B_{i, j}$ is represented by the arc from the copy of $(i, j)$ in $A_{i-1}+$ to the original $(i, j)$ in $A_{i}+$. It is important to note that each such arc includes the midpoint of $A_{i-1}+$, has length $2+\epsilon$, and includes the same points between $(i)$ and $(i+1)$ as in our previous construction.

We similarly, insert a copy of the points of $A_{i}+$ in the second half of $A_{i+1}+$. Specifically, the midpoint of $A_{i+1}+$ is a copy of $(i, 1)$, which is followed by $(i, 2), \ldots,\left(i, t_{i}-1\right),\left(i, t_{i}\right)$ with precisely the same spacing as in $A_{i}+$. With these points we can now create the arcs for the vertices in $B_{i+1}$. Specifically, using the partition $X_{i+1, j}$ as before, each $x_{j} \in X_{i+1, j}$ is represented by the arc from the original $(i, j)$ in $A_{i}+$ to the copy of $(i, j)$ in $A_{i+1}+$. It is important to note that each such arc includes the midpoint of $A_{i+1}+$, has length $2+\epsilon$, and includes the same points between $(i)$ and $(i+1)$ as in our previous construction.

We need only consider one special case to complete our construction, namely, when both $B_{i} \cup B_{i+1}$ and $B_{i} \cup B_{i-1}$ are cliques. In this case we simply map each vertex of $B_{i}$ to an arc from the midpoint of $A_{i-1}+$ to the midpoint of $A_{i}+$. This again provides arcs of length $2+\epsilon$.

Now, when $B_{i} \cup B_{i+1}$ is not a clique, this construction properly represents the edges between $B_{i}$ and $B_{i+1}$ since we simply have the same representation as before. Additionally, when $B_{i} \cup B_{i+1}$ is a clique, the arcs of $B_{i}$ and $B_{i+1}$ always include the midpoint of $A_{i}+$. This again properly represents the edges between $B_{i}$ and $B_{i+1}$. Thus, we have produced a unit circular-arc representation of $B$.

\section{$3.2 \quad$ Neighbors of Buoys}

We now generalize the results of Observation 3.1 to buoys. We examine the different types of adjacencies between vertices outside a buoy $B$ in a (pan, even hole)-free graph and vertices inside $B$. Let $x$ be a vertex of $G$ outside of $B$. We say $x$ is of type $t$ with respect to $B$ if $x$ has neighbors in exactly $t$ distinct bags $B_{i}$. It is easy to see that in a pan-free graph, $x$ cannot be of type 1 . The following lemma describes possible adjacencies between $x$ and $B$.

Lemma 3.15 Let $G$ be a (pan, even hole)-free graph. Let $B$ be an odd $\ell$-buoy of $G$ with bags $B_{0}, \ldots, B_{\ell-1}$ and let $x$ be a vertex of $G-B$ that has some neighbors in $B$.

(i) If $N(x) \cap B_{i} \neq \emptyset$, then $N(x) \cap B_{i-1} \neq \emptyset$, or $N(x) \cap B_{i+1} \neq \emptyset$. 
(ii) If $i$ and $j$ are indices such that $j \in\{2, \ldots, \ell-1\}, x$ has a neighbor in each of $B_{i}$ and $B_{i+j}$, and $x$ has no neighbors in $B_{i+1} \cup \ldots \cup B_{i+j-1}$, then $j$ is odd (i.e., the number of bags $B_{i}, \ldots, B_{i+j}$ is even).

(iii) If $x$ has neighbors in $B_{i-1}$ and neighbors in $B_{i+1}$, then $x$ is $B_{i \text {-complete. }}$

(iv) Vertex $x$ is of types 2, 3, or $\ell$. If $x$ is of type $\ell$, then $x$ is $B$-complete.

(v) If $x$ is of type 3 , then $x$ has neighbors in three consecutive $B_{i}$ s.

(vi) Suppose $x$ is of type 2 and has neighbors in $B_{i}$ and in $B_{i+1}$. Then $\{x\} \cup B_{i} \cup B_{i+1}$ is a clique.

Proof: Let $B_{i}$ be a fixed bag. Let $d_{t}$ be a dominant vertex of $B_{t}, 0 \leq t \leq \ell-1$. The vertices $d_{t}$ exist for all $t$ by Observation 3.9 .

Proof of (i). Suppose $x$ is adjacent to some $b_{i} \in B_{i}$ and is $\left(B_{i-1} \cup B_{i+1}\right)$-null. Let $b_{i-1}$ be a neighbor of $b_{i}$ in $B_{i-1}$ and let $b_{i+1}$ be a neighbor of $b_{i}$ in $B_{i+1}$. By Corollary 3.4. there is a skeleton $C$ containing $b_{i-1}, b_{i}, b_{i+1}$. But then part (i) of Observation 3.1 is contradicted.

Proof of (ii). Suppose (ii) is false. Let $a$ and $a^{\prime}$ be neighbors of $x$ in $B_{i}$ and $B_{i+j}$ respectively. Since $d_{t}$ is a dominant vertex of $B_{t}$ for all $t,\left\{x, a, d_{i+1}, \ldots, d_{i+j-1}, a^{\prime}\right\}$ induces an even hole.

Proof of (iii). Suppose there is $b_{i} \in B_{i}$ which is not a neighbor of $x$. We will distinguish among three cases: (1) $b_{i}$ and $x$ have common neighbors $a_{i-1} \in B_{i-1}$ and $a_{i+1} \in B_{i+1}$; (2) $b_{i}$ and $x$ have no common neighbors in $B_{i-1} \cup B_{i+1}$; and (3) $b_{i}$ and $x$ have a common neighbor $a_{i-1} \in B_{i-1}$, but no common neighbor in $B_{i+1}$. In case (1), $\left\{x, a_{i-1}, b_{i}, a_{i+1}\right\}$ induces a $C_{4}$. For case (2), let $a_{i-1}$ be a neighbor of $x$ in $B_{i-1}$ and let $a_{i+1}$ be a neighbor of $x$ in $B_{i+1}$. Note that $b_{i}$ must have neighbors $b_{i-1}$ in $B_{i-1}$ and $b_{i+1}$ in $B_{i+1}$. Thus, in this case, $\left\{x, a_{i-1}, b_{i-1}, b_{i}, b_{i+1}, a_{i+1}\right\}$ induces a $C_{6}$.

Now we handle case (3). Let $a_{i+1}$ be a neighbor of $x$ in $B_{i+1}$ and $b_{i+1}$ be a neighbor of $b_{i}$ in $B_{i+1}$. The dominant vertex $d_{i}$ of $B_{i}$ is adjacent to both $a_{i-1}$ and $a_{i+1}$. Thus, $d_{i}$ is adjacent to $x$, for otherwise $\left\{x, a_{i-1}, d_{i}, a_{i+1}\right\}$ induces a $C_{4}$.

Suppose $x$ is not adjacent to $d_{i-2}$. By Corollary 3.4 there is a skeleton $C$ containing $d_{i-2}, a_{i-1}, b_{i}$. But then $x$ and $C$ contradict Observation 3.1 (i). Thus $x$ is adjacent to $d_{i-2}$. Let $P$ be the path $d_{i-2} a_{i-1} d_{i} b_{i+1}$. Corollary 3.4 implies there is a skeleton $C$ containing $P$. Since $d_{i+2}$ is a dominant vertex of $B_{i+2}$, we may assume $d_{i+2} \in C\left(d_{i+2}\right.$ may replace the vertex of $\left.C \cap B_{i+2}\right)$. Since $x$ has at least three neighbors and one non-neighbor $\left(b_{i+1}\right)$ on $C$, Observation 3.1 (v) implies that $x$ has exactly three neighbors on $C$. In particular, we have $x d_{i+2} \notin E(G)$. Let $C^{\prime}=\left(C-\left\{b_{i+1}\right\}\right) \cup\left\{a_{i+1}\right\}$. Then $C^{\prime}$ is a hole. But now, $x$ has at least four neighbors and one non-neighbor $\left(d_{i+2}\right)$ on $C^{\prime}$, a contradiction to Observation 3.1 (v).

Proof of (iv). Suppose $x$ is of a type different from 2, 3, or $\ell$. There are indices $i, j$ such that $x$ has neighbors in each of $B_{i}$ and $B_{j}$ and no neighbors in $B_{i+1}, \ldots, B_{j-1}$ and $|j-i| \not \equiv 1 \bmod \ell$. By (i), $x$ has neighbors in $B_{i-1}$ and in $B_{j+1}$. By (ii), the number of sets $B_{i}, B_{i+1}, \ldots, B_{j}$ is even. So the number of sets $B_{j+1}, B_{j+2}, \ldots, B_{i-1}$ is odd. Let $b_{t}$ be a neighbor (if one exists) of $x$ in $B_{t}$, for all $t$. Consider the path $P=b_{j+1} d_{j+2} \ldots d_{i-2} b_{i-1}$. Vertex $x$ must be adjacent to an interior vertex $p$ of this path, for otherwise $P$ and $x$ form an even hole. But now there is a pan formed by the vertices $p, x, b_{i}, d_{i+1}, \ldots, d_{j-1}, b_{j}$.

If $x$ is of type $\ell$, then by (iii), $x$ is $B_{i}$-complete for all $i$. 
Proof of (v) Let $x$ be of type 3. Assume $x$ has a neighbor in $B_{i}$ for some $i$. By (i) we may assume $x$ has a neighbor in $B_{i+1}$. Let $B_{j}$ be the third bag such that $x$ has neighbors in $B_{j}$. If $j \notin\{i-1, i+2\}$, then (i) is contradicted.

Proof of (vi). Suppose $x$ is of type 2 and has neighbors in $B_{i}$ and $B_{i+1}$ and let $a_{i} \in B_{i}$ and $a_{i+1} \in B_{i+1}$ be neighbors of $x$. If $a_{i}$ and $a_{i+1}$ are not adjacent, then $\left\{a_{i}, x, a_{i+1}, d_{i+2}\right.$, $\left.\ldots, d_{i-1}\right\}$ induces an even hole. Thus, the neighbors of $x$ in $B$ form a clique.

We now show that $x$ is adjacent to every neighbor $b_{i+1} \in B_{i+1}$ of $a_{i}$. Suppose $x$ is not adjacent to $b_{i+1}$. Consider the skeleton $C$ formed by the vertices $a_{i}, b_{i+1}, d_{i+2}, d_{i+3}, \ldots, d_{i-1}$. Vertex $x$ has only one neighbor on this hole, a contradiction to part (v) of Observation 3.1. By symmetry, $x$ is adjacent to every neighbor $b_{i} \in B_{i}$ of $a_{i+1}$.

From the previous paragraph $x$ must be adjacent to both $d_{i}$ and $d_{i+1}$ since they are neighbors of $a_{i+1}$ and $a_{i}$ respectively. Thus, $x$ is adjacent to all of $B_{i} \cup B_{i+1}$ and as such $\{x\} \cup B_{i} \cup B_{i+1}$ form a clique.

From the proof of Lemma 3.15, we can extract a linear-time algorithm to establish the following lemma.

Lemma 3.16 Let $G$ be a graph. Let $B$ be an odd $\ell$-buoy of $G$ with bags $B_{0}, \ldots, B_{\ell-1}$, and let $x$ be a vertex of $G-B$ that has some neighbors in $B$. If $x$ fails to satisfy (i)-(vi) of Lemma 3.15, then $G$ contains a pan or an even hole, and such an induced subgraph can be found in linear time.

\subsection{Structure Theorem}

Now that we understand the structure of buoys (see Section 3.1) and their neighbors (see Section 3.2), we are ready to prove the structure theorem introduced in Section 2. We prove the following theorem which together with Theorem 3.14 implies Theorem 2.3. Recall that $\mathcal{C}$ is the class of graphs $G$ such that every atom of $G$ is (pan, even hole)-free.

Theorem 3.17 If $G$ is a connected graph in $\mathcal{C}$ then

(i) $G$ is a clique, or

(ii) $G$ contains a clique cutset, or

(iii) For every maximal buoy $B$ of $G$, either $B$ is a full buoy of $G$, or $G$ is the join of $B$ and a clique.

Proof: We may assume $G$ is connected and contains an odd hole $C$, for otherwise $G$ is chordal and the theorem holds. Let $\ell$ be the length of $C$. Since $G$ contains $C, G$ contains a maximal buoy $B$ with bags $B_{0}, B_{1}, \ldots, B_{\ell-1}$ and skeleton $C$ (here, as usual, "maximal" is meant with respect to set inclusion, not size). If $G-B=\emptyset$, then $G$ is a full buoy and we are done. Let $A$ be the set of vertices in $G-B$ with some neighbor in $B$, and $R$ be the set of vertices in $G$ with no neighbor in $B$. Consider a vertex $x$ in $A$. By Lemma 3.15, $x$ is of types 2,3 , or $\ell$. If $x$ is of type 3 , then, by $(i i i)$ and $(i v)$ of Lemma 3.15, $x$ has neighbors in three consecutive bags $B_{i-1}, B_{i}, B_{i+1}$ and is complete to $B_{i}$. In particular, $B \cup\{x\}$ is a larger buoy with bags $B_{0}, B_{1}, \ldots, B_{i-1}, B_{i} \cup\{x\}, B_{i+1}, \ldots, B_{\ell-1}$, a contradiction to our choice of $B$. Also, when $x$ is of type 2, then, by $(i i)$ and $(v i)$ of Lemma 3.15, there is an index $i$ such that $\{x\} \cup B_{i} \cup B_{i+1}$ is a clique. Thus, $A$ can be partitioned into sets $A_{0}, A_{1}, \ldots A_{\ell-1}, U$ such that

- $a \in A_{i}$ if and only if $N(a) \cap B=B_{i} \cup B_{i+1}$; and 
- $u \in U$ if and only if $u$ is $B$-complete.

Note that all type- $\ell$ vertices are in $U$. The set $U$ (if non-empty) induces a clique for otherwise, two non-adjacent vertices of $U$ and two non-adjacent vertices of $B$ form a $C_{4}$. We may assume there is a non-empty $A_{i}$, for otherwise $G$ is the join of $B$ and $U$ (if $R=\emptyset$ ), or $U$ is a clique cutset of $G$ separating $B$ and $R$ (if $R \neq \emptyset$ ).

Consider a non-empty set $A_{i}$ and let $D=B_{i} \cup B_{i+1} \cup U$. The set $D$ is a clique by Lemma 3.15. We will show that $D$ is a clique cutset. Suppose it is not a clique cutset. Then, in $G-D$, there is a shortest path $P$ from a vertex $a_{i} \in A_{i}$ to a vertex $b \in B-\left(B_{i} \cup B_{i+1}\right)$. Enumerate the vertices of $P$ as $v_{1}, v_{2}, \ldots, v_{t}$ with $v_{1}=a_{i}$ and $v_{t}=b$. Since the path is shortest, $v_{t-1} \in A_{j}$ for some $j \neq i, t \geq 3$, and $v_{j} \in R$ for $j \in\{2,3, \ldots t-2\}$ (when $t>3$ ). There are two induced paths whose endpoints are $v_{t-1}$ and $a_{i}$, and whose interior vertices are disjoint and lie in $B$. We may enumerate one as $P_{1}=v_{t-1} b_{j+1} \ldots b_{i} a_{i}$, and the second one as $P_{2}=v_{t-1} b_{j} \ldots b_{i+1} a_{i}$ with $b_{k} \in B_{k}$ for all $k$. Since $\ell$ is odd, $P_{1}$ and $P_{2}$ have different parities. Let $P^{\prime}=P-\left\{v_{t}\right\}$. One of the two holes induced by $P_{1} \cup P^{\prime}$ and $P_{2} \cup P^{\prime}$ has to be even, a contradiction.

An algorithm can be extracted from the proof of Theorem 3.17 to prove the following theorem.

Theorem 3.18 Let $B$ be a maximal buoy of a graph $G$. If $B$ is not a full buoy of $G$ and if $G$ is not the join of $B$ and a clique, then $G$ contains an even hole or a pan, and such an induced subgraph can be found in linear time.

\section{A coloring algorithm for (pan, even hole)-free graphs}

In this section, we discuss a polynomial-time algorithm to color a graph in $\mathcal{C}$. Consider a graph $G$ with a clique cutset decomposition tree $T(G)$. From the discussion in Section 2 , if we can color the atoms of $G$ in polynomial time, then we can also color $G$. The purpose of this section is to show that $G$ can indeed be colored in polynomial time.

In [28], an $O\left(n^{2}\right)$-time algorithm is given for coloring proper circular-arc graphs. For unit circular-arc graphs, this can be improved. First, we use the $O(n+m)$-time algorithm of [24] for recognizing unit circular-arc graphs to construct a unit circular-arc representation. Then we use the $O\left(n^{1.5}\right)$-time algorithm of [31] to find a minimum coloring of a unit circular-arc graph given the representation. This gives an $O\left(n^{1.5}+m\right)$-time algorithm to color unit circular-arc graphs.

Thus, from Theorems 3.14 and 3.17 , we have the following two results.

Theorem 4.1 There is an $O\left(n^{1.5}+m\right)$-time algorithm to find a minimum coloring of a (pan, even hole)-free graph that is either a buoy or the join of a buoy and a clique.

Proof: Let $G$ be a (pan, even hole)-free graph that is the join of a clique $K$ and a buoy $B$. Then we have $\chi(G)=|K|+\chi(B)$. Thus, we only need to establish the theorem for (pan, even hole)-free buoys. Now the result follows from Theorem 3.14 and the $O\left(n^{1.5}+m\right)$-time algorithm to color unit circular-arc graphs.

Theorem 4.2 There is an $O\left(n^{2.5}+n m\right)$-time algorithm to find a minimum coloring of a graph in $\mathcal{C}$.

Proof: By the discussion above and the fact that the clique cutset decomposition provides at most $n-1$ atoms, we only need show there is an $O\left(n^{1.5}+m\right)$-time algorithm to color a (pan, even hole)-free atom $G$. By Theorem 2.3, $G$ is one of the following: a clique, a buoy, 
or the join of a clique and a buoy. Thus, by Theorem 4.1, $G$ can be optimally colored in $O\left(n^{1.5}+m\right)$ time.

\section{Recognition algorithms for (pan, even hole)-free graphs}

In this section, we give two polynomial-time algorithms to recognize (pan, even hole)-free graphs. We note that a polynomial-time algorithm for recognizing (pan, even hole)-free graphs can easily be converted to a polynomial-time algorithm for recognizing graphs in $\mathcal{C}$.

There exist several polynomial-time algorithms $([7,8,9])$ for finding an even hole in a graph. But the fastest such algorithm [7] runs in time $O\left(n^{5} m^{3}\right) \leq O\left(n^{11}\right)$. A straightforward algorithm to recognize a (pan, even hole)-free graph is to test for a pan using Theorem 5.3 below, and then to test for an even hole. In particular, we can recognize (pan, even hole)-free graphs in $O\left(n^{5} m^{3}\right)$ time. We will design faster algorithms for (pan, even hole)-free graph recognition. We provide two recognition algorithms. The first uses the fact that the (pan, even hole)-free atoms are unit circular-arc graphs and recognizes (pan, even hole)-free graphs in $O\left(n m^{2}+n^{2} m \log \log n\right)$ time. The second uses the fact that the atoms are essentially very special buoys and runs in $O(n m)$ time.

Similar to our coloring algorithm, we note that detecting an even hole in a graph $G$ is easily reduced to checking for an even hole in an atom. That is, suppose a graph $G$ has a clique cutset $C$ and consider the subgraphs $G_{1}=G\left[V_{1}\right]$ and $G_{2}=G\left[V_{2}\right]$ where $V=V_{1} \cup V_{2}$ and $C=V_{1} \cap V_{2}$. Then $G$ contains an even hole if and only if $G_{1}$ or $G_{2}$ does; i.e., when testing for even holes one need only consider atoms.

As we have mentioned previously, the clique cutset decomposition tree $T(G)$ can be computed in $O(n m)$ time such that there are fewer than $n$ atoms 33 .

\subsection{Recognition via testing for pans and then testing for even holes in unit circular-arc atoms}

We will first describe an algorithm to find a pan in a graph.

Lemma 5.1 Let a graph $G$ be an atom. Then every vertex $v$ of $G$ is universal, or lies in a hole of $G$. Furthermore, there is a linear-time algorithm to find a hole containing $v$ when $v$ is not universal.

Proof: Let $G$ be an atom and $v$ be a vertex of $G$. Let $M(v)=V(G)-(N(v) \cup\{v\})$. If $M(v)=\emptyset$, then $v$ is universal. Compute the components $C_{1}, \ldots, C_{t}$ of $G[M(v)]$. For each $C_{i}$, compute the set $N_{i}$ of vertices in $N(v)$ that have some neighbors in $C_{i}$. If some $N_{i}$ is a clique, then $N_{i}$ is a clique cutset separating $v$ from $C_{i}$, a contradiction. Thus, none of the $N_{i}$ s are cliques. Choose an arbitrary set $N_{i}$. Consider two non-adjacent vertices $x$ and $y$ in $N_{i}$. Find a chordless path $P$ from $x$ to $y$ whose interior vertices lie entirely in $C_{i}$. Then $P$ and $v$ induce a hole in $G$.

Lemma 5.2 Given a graph $G$ and a vertex $v$ in $G$, there is an $O(n m)$ time algorithm to find a hole containing $v$, if such a hole exists.

Proof: Construct in $O(\mathrm{~nm})$ time the clique cutset decomposition $T(G)$ of $G$. Consider all the atoms of $T(G)$ containing $v$. If $v$ is a universal vertex in all such atoms, then $v$ does not lie on any hole of $G$. Suppose $v$ is not universal in some atom $A$. By Lemma 5.1, we can find a hole containing $v$ in linear time. 
Theorem 5.3 There is an $O\left(n m^{2}\right)$-time algorithm to find a pan in a graph, if one exists.

Proof: For an edge $a b$ we can check, by Lemma 5.2 in $O(n m)$ time whether $a b$ is the handle of a pan by finding a hole containing $a$ (respectively, $b$ ) in the subgraph of $G$ induced by $V(G)-(N(b)-\{a\})$ (respectively, $V(G)-(N(a)-\{b\})$.) Since $G$ has $m$ edges, the time bound of the theorem follows.

Now, to recognize whether $H$ is a (pan, even hole)-free graph, we first use Theorem 5.3 to test for a pan. If $H$ has no pan, find the clique cutset decomposition.

For an atom $G$, Theorem 2.3 implies that $G$ is either a unit circular-arc graph or the join of a clique $K$ and a unit circular-arc graph $G^{\prime}$. In the latter case, $G$ is even-hole-free if and only if $G^{\prime}$ is even-hole-free. One can test whether a graph is a unit circular-arc graph in linear time [24]. In particular, if $G^{\prime}$ is not unit circular-arc, then we know $G$ must have an even hole (by Theorem 3.14). Additionally, an $O(n m \log \log n)$-time algorithm is known for finding an even (or odd) hole in a circular-arc graph [6]. That is, via the clique cutset decomposition, we can test whether a graph in $\mathcal{C}$ contains an even hole in $O\left(n^{2} m \log \log n\right)$ time (since the decomposition can be computed in $O(n m)$ time and has $O(n)$ atoms).

Thus, for a given graph $H$, we can recognize whether $H$ is (pan, even hole)-free in $O\left(n m^{2}+n^{2} m \log \log n\right)$ time.

\subsection{Recognition via buoy construction}

We now present an algorithm which relies on the buoy structure of a (pan, even hole)-free graph to test whether an atom is (pan, even hole)-free, and if it is not, to find a pan or even hole. Recall that, by Theorem 3.17, in a (pan, even hole)-free atom $G$ either every maximal buoy is a full buoy or $G$ is the join of a clique and a buoy. With this approach, we do not attempt to directly find a pan. Instead, a pan (if it exists) can be found by examining the buoys and their neighborhoods.

An atom $A$ of graph $G$ is maximal if any induced subgraph $H$ of $G$ that properly contains $A$ is not an atom, i.e., if $H$ has a clique cutset. The atoms produced by the clique cutset decomposition are maximal.

The algorithm will produce a forbidden induced subgraph, if one exists. The algorithm has three steps.

(1) Find a clique cutset decomposition tree $T(G)$ of $G$.

(2) For each (maximal) atom $A$ of $T(G)$, (i) extract a forbidden induced subgraph (if one exists) from $A$, or (ii) show that $A$ is a buoy, or (iii) find a partition of the vertices of $A$ into the join of a buoy and a clique. The involved buoy will satisfy Observation 3.9 .

(3) For each atom $A$ of $T(G)$, verify that no holes of $A$ form a pan with a vertex outside $A$.

We will show that steps (2) and (3) can be done in linear time for an atom. This shows the algorithm runs in $O(n m)$ time.

The correctness of step (2) follows from the following theorem.

Theorem 5.4 Let $G$ be an atom. There is a linear-time algorithm to output

(i) a pan, or

(ii) an even hole, or 
(iii) a certificate that $G$ is (pan, even hole)-free, and either a certificate that $G$ is a buoy or a partition of $V(G)$ into sets $B$ and $K$ such that $B$ is a buoy, $K$ is a clique, and $G$ is the join of $B$ and $K$.

To prove Theorem 5.4, we will need the following three lemmas.

Lemma 5.5 If $B$ is an $\ell$-buoy where each $B_{i}$ can be ordered by neighborhood inclusion, then every hole in $B$ has length $\ell$.

Proof: Consider a hole $H$ of $B$. No two vertices of $H$ have comparable neighborhoods. Thus, each bag of $B$ contains at most one vertex of $H$. If $H$ has fewer than $\ell$ vertices, then $H$ is not a hole (a contradiction) since vertices in a bag can only have neighbors in the bag preceding it and the bag following it in the cyclic order. So $H$ has length $\ell$.

Lemma 5.6 Let $B$ be an odd $\ell$-buoy where each bag $B_{i}$ can be ordered by neighborhood inclusion. The following three statements are equivalent

(i) There are two vertices $a, b$ in some $B_{i}$ such that a strictly dominates $b$ in $B_{i-1}$, but $b$ does not dominate $a$ in $B_{i+1}$.

(ii) B has a pan.

(iii) There is a subscript $i$ such that $B_{i-1} \cup B_{i}$ and $B_{i} \cup B_{i+1}$ are both not cliques.

Proof: First, note that Lemma 5.5 implies that $B$ has no even hole. Now the fact that (i) $\Longrightarrow$ (ii) follows from Observation 3.12 . Next, we prove the implication (ii) $\Longrightarrow$ (iii). Suppose $B$ contains a pan. By Lemma 5.5, the hole of this pan must contain exactly one vertex of each bag. Let the pan consist of vertices $a, b_{0}, b_{1}, \ldots, b_{\ell-1}$ where $b_{i} \in B_{i}$ and the vertices $b_{i}$ form a hole. Without loss of generality, we may assume $a \in B_{1}$. So, (iii) is satisfied with $i=1$. Finally, we prove the implication (iii) $\Longrightarrow$ (i). Suppose $B_{0} \cup B_{1}$ and $B_{1} \cup B_{2}$ are both not cliques. Let $a$ be the dominant vertex of $B_{1}$, and $b$ be the vertex in $B_{1}$ that is dominated by every other vertex of $B_{1}$. If $b$ is adjacent to every vertex in $B_{0}$, then every vertex in $B_{1}$ is adjacent to every vertex in $B_{0}$, a contradiction. So $b$ is non-adjacent to some vertex of $B_{0}$, i.e., $a$ strictly dominates $b$ in $B_{0}$. A symmetric argument shows that $a$ strictly dominates $b$ in $B_{2}$.

Lemma 5.7 Let $B$ be an $\ell$-buoy with bags $B_{0}, \ldots, B_{\ell-1}$ for some $\ell$. There is a linear-time algorithm to verify that the bags of $B$ admit a domination order, i.e., the vertices of each $B_{i}$ are pairwise comparable.

Proof: For each bag $B_{i}$, we order its vertices by non-decreasing size of their neighborhoods; i.e., $B_{i}$ is ordered as $b_{i}^{0}, \ldots, b_{i}^{k_{i}-1}$, where $\left|N\left(b_{i}^{0}\right)\right| \leq\left|N\left(b_{i}^{1}\right)\right| \leq \ldots \leq\left|N\left(b_{i}^{k_{i}-1}\right)\right|$ with $k_{i}=\left|B_{i}\right|$. This can be done in $O\left(\left|B_{i-1}\right|+\left|B_{i}\right|+\left|B_{i+1}\right|\right)$ time via bucket-sort. That is, sorting all of the bags can be done in $O(n)$ time. We then check that for every $j \in\left\{1, \ldots, k_{i}-1\right\}$, every neighbor of $b_{i}^{j-1}$ is a neighbor of $b_{i}^{j}$ (if this is not the case, then $b_{i}^{j-1}$ is incomparable with $b_{i}^{j}$ ). For each bag $B_{i}$, this neighborhood checking can be performed in $O\left(\sum_{j=0}^{k_{i}-1}\left|N\left(b_{i}^{j}\right)\right|\right)$ time. In particular, all such checking can be performed in $O(m)$ time. Thus, we can check that the bags of $B$ admit a domination order in $O(n+m)$ time.

Now we can prove Theorem 5.4 .

Proof: Suppose that $G$ is an atom. Using the linear-time algorithm in [32], we either confirm that $G$ is chordal (and hence is (pan, even hole)-free) or obtain a hole $H$. We may 
assume $H$ is an odd hole. We first briefly describe the algorithm. We will construct a maximal buoy $B$ with $H$ as its skeleton in $O(n+m)$ time. During this process, we verify that $B$ has the domination property of Observation 3.9 or $G$ contains a pan or even hole. If $H \neq G$ and $G$ is not the join of $B$ and a clique, then we will find a pan or even hole.

We now describe our construction of a maximal $\ell$-buoy $B$ in an atom $G$ from a hole $H=\left\{h_{0}, \ldots, h_{\ell-1}\right\}$ of $G$. We start with the initial $\ell$-buoy $B$ with bags $B_{0}=\left\{h_{0}\right\}, \ldots$, $B_{\ell-1}=\left\{h_{\ell-1}\right\}$.

Let $K$ be the set of universal vertices of $G$. We remove vertices in $K$ from $G$ since these cannot be part of an even hole or a pan. Since $K$ is not a clique cutset of $G$, removing $K$ does not make the resulting graph disconnected. Also, if $C$ is a clique cutset of $G_{K}$, then $C \cup K$ is a clique cutset of $G$. Thus, the graph we obtain by removing $K$ is still an atom.

Consider a vertex $x$ in $G-B$ with some neighbors in $B$. If $x$ is of type $t$ with $t \notin\{2,3, \ell\}$, then by Lemma 3.15, we know $G$ has a pan or even hole, and we can find this forbidden induced subgraph by Lemma 3.16 . So, $x$ is of type 2,3 or $\ell$. The only candidates to be added to $B$ are type 3 vertices. Suppose $x$ is of type 3. By Lemma 3.15, either $x$ is adjacent to three consecutive bags $B_{i-1}, B_{i}, B_{i+1}$ of $B$, or $G$ contains a pan or an even hole. Suppose $x$ is adjacent to three consecutive bags $B_{i-1}, B_{i}, B_{i+1}$. If $x$ is not adjacent to all vertices of $B_{i}$, then by Lemmas 3.15 and 3.16 , we will find a forbidden induced subgraph. Now $x$ is adjacent to all vertices of $B_{i}$. We then add $x$ to $B_{i}$. We summarize the operations described in the above paragraph with Algorithm 1, named ENGLARGE and given below.

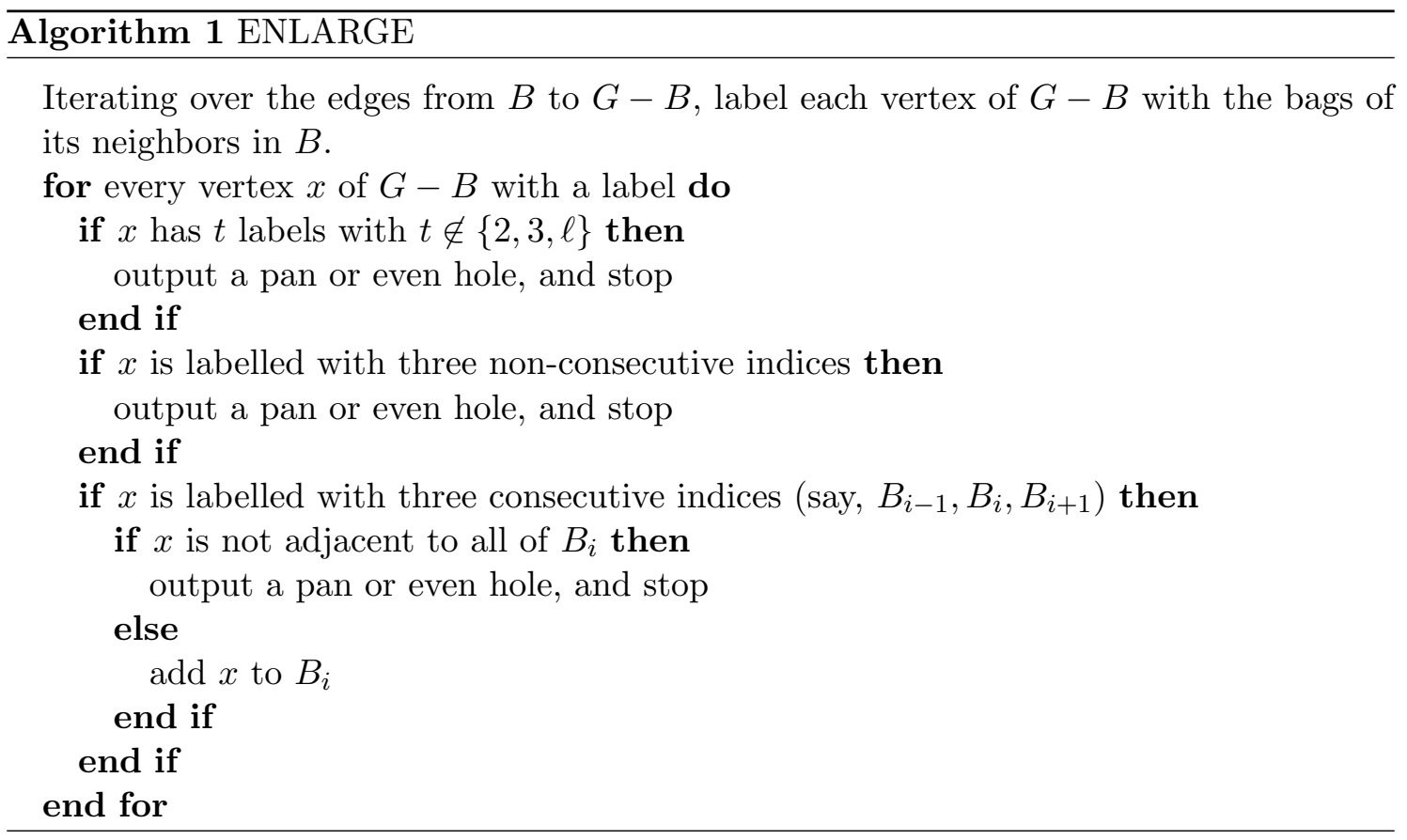

Starting with our first buoy $B$ which is an odd hole, we call ENLARGE on $B$ twice. We will show that after two calls to ENLARGE, we can decide whether $G$ is (pan, even hole)-free. After the first (respectively, second) call to ENLARGE, let $B^{1}$ (respectively, $B^{2}$ ) be the resulting buoy, and let the bags of $B^{1}$ (respectively, $B^{2}$ ) be $B_{0}^{1}, B_{1}^{1}, \ldots, B_{\ell-1}^{1}$ (respectively, $B_{0}^{2}, B_{1}^{2}, \ldots, B_{\ell-1}^{2}$ ). Note that $B_{i}^{1} \subseteq B_{i}^{2}$ for all $i$. Using Lemma 5.7, we verify in linear time that both $B^{1}$ and $B^{2}$ have the desired domination property, or else we find a pan or even hole.

Suppose there is a vertex of $G$ not belonging to any $B_{i}^{2}$. Consider a vertex $x$ in $G-B^{2}$ 
with neighbors in some of the bags. If $x$ is of type $t$ with $t \notin\{2,3, \ell\}$, then by Lemma 3.16 , we can produce a pan or even hole in linear time.

We will prove that $x$ is of type 2 or $\ell$. Suppose $x$ is of type 3. If $x$ does not have neighbors in three consecutive bags, then by Lemma 3.16, we can produce a pan or even hole in linear time. So $x$ has neighbors in three consecutive bags, say, $B_{i-1}^{2}, B_{i}^{2}, B_{i+1}^{2}$. Vertex $x$ is adjacent to all of $B_{i}^{2}$, for otherwise, by Observation 3.16 we can find a pan or even hole. So, $B^{3}=B^{2} \cup\{x\}$ is a buoy with bags $B_{0}^{2}, \ldots, B_{i-1}^{2}, B_{i}^{2} \cup\{x\}, B_{i+1}^{2}, \ldots, B_{\ell-1}^{2}$ . We may assume $B^{3}$ has the domination property of Observation 3.9 , for otherwise by Observation 3.10, we will find a pan or even hole. Thus, each bag $B_{j}^{2}$ of $B^{3}$ has a dominant vertex $d_{j}$. Since the vertex $d_{j}$ is adjacent to three vertices of $H, d_{j}$ is added to the buoy $B^{1}$ in the first iteration. Vertex $x$ is adjacent to $d_{i-1}, h_{i}, d_{i+1}$, so $x$ would have been added to $B^{2}$ in the second iteration, a contradiction.

So $x$ is of type 2 or $\ell$. (From now on, we only refer to the buoy produced after the second call; so to simplify notation, we will let $B=B^{2}$.) When $x$ is of type 2 , then, by (vi) of Lemma 3.15, there is an index $i$ such that $\{x\} \cup B_{i} \cup B_{i+1}$ is a clique, or else we can produce a pan or even hole. Thus, by $(i v)$ and (vi) of Lemma 3.15, $G-B$ can be partitioned into sets $A_{0}, A_{1}, \ldots A_{\ell-1}, U, R$ such that

- $a \in A_{i}$ if and only if $N(a) \cap B=B_{i} \cup B_{i+1}$,

- $u \in U$ if and only if $u$ is $B$-complete,

- $r \in R$ if and only if $r$ is $B$-null.

We may now use the proof of Theorem 3.17 to find a pan or even hole. The set $U$ (if non-empty) induces a clique for otherwise, two non-adjacent vertices of $U$ and two nonadjacent vertices of $B$ form a $C_{4}$. If all sets $A_{i}$ are empty, then $U$ is a clique cutset of $G$. So, some $A_{i}$ is non-empty. Since $G$ is an atom, $U \cup B_{i} \cup B_{i+1}$ is not a clique cutset separating $A_{i}$ from $B-\left(B_{i} \cup B_{i+1}\right)$. Thus, there is a shortest path $P$ from a vertex $a_{i} \in A_{i}$ to a vertex $a_{j} \in A_{j}(i \neq j)$ whose interior vertices lie entirely in $G-(B \cup U)$. Find an induced path $P^{\prime}$ with the same parity as $P$ from $a_{i}$ to $a_{j}$ whose interior vertices belongs to $B$. Then $P \cup P^{\prime}$ is an even hole.

Thus, after two calls to ENLARGE, we have constructed a full buoy $B$ of the graph $G-K$, where $K$ is the set of universal vertices we remove before the first call to ENLARGE.

To complete the proof, we only need to find a pan in $B$, if one exists. By Lemma 5.6, $B$ has no pan if and only if for every $i, B_{i-1} \cup B_{i}$ or $B_{i} \cup B_{i+1}$ is a clique. This condition can be checked in $O(m)$ time. If the condition fails for some $i$, then the proof of Lemma 5.6 shows that the dominant vertex $a$ of $B_{i}$ strictly dominates the vertex $b \in B_{i}$ with the smallest degree; and so we can find a pan using Observation 3.12 . If $K$ (the set of universal vertices of $G$ ) is non-empty, then $G$ is the join of the buoy $B$ and $K$; otherwise, $B$ is a full buoy of $G$.

Now we show that step (3) of our algorithm can be implemented in $O(n m)$ time. At this point, we know that the (maximal) atom $A$ of $T(G)$ under consideration is (pan, even hole)-free, and that $A$ is either a buoy or the join of a buoy and a clique. We need to determine that no hole of $A$ forms a pan with a vertex in $G-A$; we call such a pan straddling. We need to find a straddling pan with respect to $A$ (if one exists). An atom $A$ of a graph $G$ is maximal if for any induced subgraph $H$ of $G$ containing $A$, either $H$ has a clique cutset, or $A$ is a component of $H$. The atoms produced by the clique cutset decomposition are maximal. We will need the following observation.

Observation 5.8 If $A$ is a maximal atom of a graph $G$, then for every vertex $x$ in $G-A$, $N_{A}(x)$ is either empty or a clique. 
Proof: Suppose $N_{A}(x)$ is not empty but is not a clique. Write $G^{\prime}=G[A \cup\{x\}]$. Since $A$ is a maximal atom, $G^{\prime}$ is not an atom, i.e., $G^{\prime}$ has a clique cutset $C$. If $x \in C$, then $C-\{x\}$ is a clique cutset of $A$, a contradiction. So, $x$ belongs to a component $P$ of $G^{\prime}-C$. Vertex $x$ cannot be the only vertex of $P$, for otherwise, $N_{A}(x)$ is a subset of $C$, and therefore a clique, a contradiction. But now $C$ is a clique cutset of $A$, a contradiction.

Now consider an atom $A$ of $T(G)$ that is either a buoy $B$ or the join of a buoy $B$ and a clique $K$. We are going to describe a way to find a straddling pan (if one exists) whose hole belongs to $B$. (Vertices of $K$ do not belong to a hole in $A$.) Let the bags of $B$ be $B_{0}, B_{1}, \ldots, B_{\ell-1}$. Remember that $A$ is (pan, even hole)-free, so by Observation 3.9, each bag can be ordered by neighborhood inclusion, so by Lemma 5.5, every hole in $B$ has length $\ell$. Compute the set $Q$ of vertices of $G-A$ that have neighbors in $B$. The set $Q$ can be computed in $O(m)$ time. Let $Q_{i}$ be the set of vertices $x$ of $Q$ with $N_{B}(x) \cap B=B_{i} \cup B_{i+1}$. Since $A$ is a maximal atom, the graph $G[A \cup Q]$ contains a clique cutset $C$ such that $C \subset A$. It follows from Observation 5.8 that, with respect to the buoy $B$, every vertex in $Q$ is of type 1 or 2. Furthermore, since $A$ is (pan, even hole)-free, it follows from Lemma 3.15(vi) that every vertex of type 2 belongs to some $Q_{i}$. If some $x \in Q$ is of type 1 , then clearly a straddling pan can be found in linear time. Now, every vertex $x$ in $Q_{i}$ is such that $\{x\} \cup B_{i} \cup B_{i+1}$ is a clique, and we conclude there is no straddling pan.

Thus, for a maximal atom, we can determine in $O(m)$ time whether a straddling pan exists. Since there are at most $n-1$ atoms of $T(G)$, we can implement step 3 in $O(n m)$ time. This completes the proof of Theorem 2.4.

Theorem 2.4 Given a graph $G$, a pan or even hole of $G$, if one exists, can be found in $O(n m)$ time.

Note that for an input graph $G$, if $G$ is not (pan, even hole)-free, our algorithm produces a pan or an even hole. If $G$ is (pan, even hole)-free, the algorithm produces a clique cutset decomposition tree which satisfies Theorem 5.9 below; furthermore, the set of atoms of every clique cutset decomposition tree will satisfy (i) and (ii) below.

Theorem 5.9 A graph $G$ is (pan, even hole)-free if and only if there is a clique cutset decomposition tree with most $n-1$ atoms $G_{j}$ such that

(i) Each atom $G_{j}$ is either a clique or consists of a buoy $B\left(G_{j}\right)$ and a possibly empty set $U_{j}$ of universal vertices; the buoy $B\left(G_{j}\right)$ has an odd number of bags; each bag can be ordered by neighborhood inclusion; and, for each consecutive triple of bags either the first two or second two form a clique.

(ii) Further, for each atom $G_{j}$ which is not a clique, the neighborhood of $V\left(G_{j}\right)$ in $G$ can be partitioned into sets $A_{i}$, some of which may be empty, where $A_{i}$ is universal to the ith and $(i+1)$ st bags of the buoy $B\left(G_{j}\right)$ and $A_{i}$ has no other neighbors in $B\left(G_{j}\right)$.

The correctness of the algorithm proves the "only if" part of the theorem. To see that the "if" part holds, note that a graph $G_{j}$ satisfying property (i) of Theorem 5.9 is (pan, even hole)-free. Since any hole of $G$ must lie in some atom $G_{j}$, property (ii) then guarantees that there is no straddling pan whose hole is in $G_{j}$.

It follows from Theorem 5.9 that our algorithm is certifying. The certificate given by Theorem 5.9 has size $O(n m)$. 


\section{Tree-width and $\chi$-boundedness}

In this section we bound the tree-width of (pan, even hole)-free graphs in terms of their clique number (see Theorem 6.4). This bound immediately provides a bound on the chromatic number (see Corollary 6.5).

A tree decomposition $(T, t)$ of a graph $G$ is defined to be a tree $T$ together with a function $t: V(G) \rightarrow V(T)$ such that:

- For every $v \in V(G), t(v)$ induces a subtree of $T$.

- For every $u v \in E(G), t(v) \cap t(u) \neq \emptyset$.

Notice that a simple tree decomposition of any graph can be obtained by choosing $T$ to be a single vertex and mapping every vertex of $G$ to this vertex. The vertices of $T$ are often treated as sets, referred to as the bags of the tree decomposition, and the elements of a bag $b$ are the vertices $v$ of $G$ where $b \in t(v)$. For a tree decomposition $(T, t)$ of a graph $G$, the tree-width of $(T, t)$, denoted $t w(T, t)$, is the size of the largest bag of $T$ minus 1 ; i.e., $t w(T, t)=\max _{b \in T}(|b|-1)$. For a graph $G$, the tree-width of $G$, denoted $t w(G)$, is the smallest tree-width of any tree decomposition of $G$.

We use the following three well-known and easy results regarding tree-width.

Observation 6.1 For a graph $G, \chi(G) \leq t w(G)+1$.

Proof: Let $(T, t)$ be a tree decomposition of $G$ with $t w(T, t)=t w(G)$. We create a supergraph $G^{\prime}$ by completing the bags of $(T, t)$ to cliques. This resulting graph is chordal, and thus perfect. So we now have $\chi(G) \leq \chi\left(G^{\prime}\right)=\omega\left(G^{\prime}\right)=t w(G)+1$.

Lemma 6.2 If $G$ contains a clique cutset $S$ where $G_{1}, \ldots, G_{k}$ are the components of $G-S$, then:

$$
t w(G)=\max _{i} t w\left(G\left[G_{i} \cup S\right]\right)
$$

Lemma 6.3 Let $G$ be a graph that is the join of a graph $B$ and a clique $K$. Then

$$
t w(G)=t w(B)+|K|
$$

where $|K|$ denotes the number of vertices of $K$.

For further information on tree-width, see [30].

Theorem 6.4 A (pan, even hole)-free graph $G$ has $t w(G)+1 \leq 1.5 \omega(G)$.

Proof: By Lemmas 6.2 and 6.3 and Theorem 3.17, we only need show that $t w(B) \leq$ $1.5 \omega(B)$ for any buoy in $G$. Recall that, for every bag $B_{i}$ of $B$, either $B_{i} \cup B_{i+1}$ is a clique or $B_{i-1} \cup B_{i}$ is a clique. In particular, we can build a tree representation $(T, t)$ of $B$ where $T$ is path using the unit circular-arc construction from the proof of Theorem 3.14 . To do this we choose the smallest $B_{i}$, and "split" the unit circular-arc representation at the point $(i)$ and "unroll" it onto a line. We now have a path where every point from our unit circular-arc representation is a bag, and the extreme bags are copies of the bag corresponding to the point $(i)$. Thus, by adding the vertices of $B_{i}$ to every bag on this path, we obtain a tree representation of $B$. It is easy to see that the largest bag in this representation has size $\omega(G)+\left|B_{i}\right| \leq 1.5 \omega(G)$. 
Theorem 6.4 is tight since odd cycles have tree-width two and clique number two. Similarly, by making a buoy with an odd number of bags such that each bag has $k$ vertices and $B_{i} \cup B_{i+1}$ is a clique for every $i$, we have a graph whose tree-width is $3 k-1$ and whose clique number is $2 k$. (See Figure 2 for an example with $k=2$.) Moreover, by Observation 6.1. we obtain the following corollary.

Corollary 6.5 A (pan, even hole)-free graph $G$ has $\chi(G) \leq 1.5 \omega(G)$.

\section{Conclusion and open problems}

In this paper, we studied the structure of (claw, even hole)-free graphs. It turned out that our results apply to the larger class of (pan, even hole)-free graphs. From the structure results, we obtained fast recognition and coloring algorithms for (pan, even hole)-free graphs. The complexity of coloring even-hole-free graphs is unknown. It follows from Corollary 1 in [23] that coloring odd-hole-free graphs is NP-Complete. Thus, the following problem, analogous to our result, is of interest to us.

Problem 7.1 What is the complexity of coloring (pan, odd hole)-free graphs?

Observation 6.1 shows the tree-width of a (pan, even hole)-free graphs is bounded by a function in the clique number. It is conceivable that a more general statement holds.

Problem 7.2 Is the tree-width of an even-hole-free graph bounded by a function of its clique number?

The clique-width of a graph $G$, denoted by $c w(G)$, is the minimum number of labels needed to construct $G$ using the following four operations:

(i) Creation of a new vertex $v$ with label $i$.

(ii) Disjoint union of two labeled graphs.

(iii) Joining each vertex with label $i$ to each vertex with label $j$.

(iv) Changing label $i$ to $j$.

It is known [10] that for any graph $G, c w(G) \leq 3 \cdot 2^{t w(G)-1}$ and that [12] $c w(\bar{G}) \leq 2 \cdot c w(G)$ where $\bar{G}$ is the complement of $G$.

In [11, it is shown that every problem definable in a certain kind of Monadic Second Order Logic, called $\operatorname{LinEMSOL}\left(\tau_{1}, L\right)$ is linear-time solvable on any graph class with bounded clique-width for which a $k$-expression can be constructed in linear time. In [11], it is mentioned that, roughly speaking, $\operatorname{MSOL}\left(\tau_{1}\right)$ is Monadic Second Order Logic with quantification over subsets of vertices but not of edges; $\operatorname{MSOL}\left(\tau_{1}, L\right)$ is the restriction of $\operatorname{MSOL}\left(\tau_{1}\right)$ with the addition of labels added to the vertices, and $\operatorname{LinEMSOL}\left(\tau_{1}, L\right)$ is the restriction of $\operatorname{MSOL}\left(\tau_{1}, L\right)$ which allows search for sets of vertices which are optimal with respect to some linear evaluation functions. The problems Vertex Cover, Maximum Weight Stable Set, Maximum Weight Clique, Steiner Tree and Domination are examples of $\operatorname{LinEMSOL}\left(\tau_{1}, L\right)$ definable problems. Furthermore, from the results of [29] and [22], it follows that the chromatic number of any class of graphs with bounded clique-width can be computed in polynomial time.

In [25], it is shown that split graphs have unbounded clique-width. It follows that even-hole-free graphs have unbounded clique-width. However, it might be possible that the clique-width of an even-hole-free graph is bounded by a function of its clique number. To conclude our paper, we pose this as an open problem. 
Problem 7.3 Is the clique-width of an even-hole-free graph bounded by a function of its clique number?

\section{References}

[1] P. Aboulker, P. Charbit, N. Trotignon and K. Vušković. Vertex elimination orderings for hereditary graph classes. Discrete Mathematics 338 (2015), no. 5, pp. 825-834.

[2] L. Addario-Berry, M. Chudnovsky, F. Havet, B. Reed, and P. Seymour. Bisimplicial vertices in even-hole-free graphs. Journal of Combinatorial Theory Series B 98 (2008), pp. 1119-1164.

[3] C. Berge and V. Chvátal (eds.). Topics on Perfect Graphs. North-Holland, Amsterdam, 1984.

[4] A. Brandstat, V. Lozin, and R. Mosca. Independent sets of maximum weight in panfree graphs. SIAM Journal on Discrete Mathematics 34 (2010), pp. 239-254.

[5] P. Buneman. A characterization of rigid circuit graphs. Discrete Mathematics 9 (1974), pp. 205-212.

[6] K. Cameron, E. Eschen, C. T. Hoàng, and R. Sritharan. Recognition of perfect circular-arc graphs. In: Graph Theory in Paris, A. Bondy, J. Fonlupt, J.-L. Fouquet, J.-C. Fournier, and J. L. Ramirez Alfonsin (eds.), pp. 97-108. Birkhauser, Basel, 2007.

[7] H.-C. Chang, and H.I. Lu. A faster algorithm to recognize even-hole-free graphs. $J$. Combin. Theory Ser. B 113 (2015), pp. 141-161.

[8] M. Chudnovsky, K. Kawarabayashi, and P. Seymour. Detecting even holes. J. Graph Theory 48 (2005), pp. 85-111.

[9] M. Conforti, G. Cornuejols, A. Kapoor, and K. Vušković. Even-hole-free graphs part II: Recognition algorithm. J. Graph Theory 40 (2002), pp. 238-266.

[10] D. G. Corneil and U. Rotics. On the relationship between clique-width and treewidth. SIAM J. Comput. 34 (2005), pp. 825-847.

[11] B. Courcelle, J.A. Makowsky, and U. Rotics. Linear time solvable optimization problems on graphs of bounded clique width. Theory of Computing Systems 33 (2000), pp. $125-150$.

[12] B. Courcelle and S. Olariu. Upper bounds to the clique-width of a graph. Discrete Applied Math. 101 (2000), pp. 77-114.

[13] M.V.G. da Silva and K. Vušković. Triangulated neighborhoods in even-hiole-free graphs. Discrete Mathematics 307 (2007), pp. 1065-1073.

[14] G. A. Dirac. On rigid circuit graphs. Abh. Math. Sem. Univ. Hamburg 25 (1961), pp. $71-76$.

[15] C. De Simone. On the vertex packing problem. Graphs Combin. 9 (1993), no. 1, pp. $19-30$. 
[16] F. Gavril. The intersection graphs of subtrees of trees are exactly the chordal graphs. J. Combin. Theory Ser. B 16 (1974), pp. 47-56.

[17] F. Gavril. Algorithms for minimum coloring, maximum clique, minimum covering by cliques, and maximum independent set of a chordal graph. SIAM Journal on Computing 1 (1972), pp. 180-187.

[18] M. C. Golumbic. Algorithmic Graph Theory and Perfect Graphs, Academic Press, New York, 1980.

[19] I. Holyer, The NP-completeness of edge-coloring. SIAM J. Computing 10 (1981), pp. $718-720$.

[20] T. Kloks, D. Kratsch, and H. Müller. Finding and counting small induced subgraphs efficiently. Information Processing Letters 74:3 (2000), pp. 115-121.

[21] T. Kloks, H. Müller, and K. Vušković. Even-hole-free graphs that do not contain diamonds: a structure theorem and its consequences. J. Combin. Theory Ser. B 99 (2009), no. 5, pp. 733-800.

[22] D. Kobler and U. Rotics, Edge dominating set and colorings on graphs with fixed clique-width. Discrete Applied Mathematics 126 (2003), pp. 197-221.

[23] J. Kratochvil, D. Kral, Zs. Tuza, and G.J. Woeginger, Complexity of coloring graphs without forbidden induced subgraphs. WG 2001, Lecture Notes in Computer Science, Vol. 2204, Springer, Berlin, 2001, pp. 254-262.

[24] M. C. Lin and J. L. Szwarcfiter. Unit circular-arc representations and feasible circulations. SIAM J. Discrete Math. 22 (2008), no.1, pp. 409-423.

[25] J.A. Makowsky and U. Rotics. On the clique-width of graphs with few $P_{4}$. Internat. J. Found. Comput. Sci. 10 (1999), pp. 329-348.

[26] S. E. Markossian, G. S. Gasparian, and B. A. Reed. $\beta$-perfect graphs. J. Combin. Theory Ser. B 67 (1996), no. 1, pp. 1-11.

[27] S. Olariu. The Strong Perfect Graph Conjecture for pan-free graphs. J. Combin. Theory B 47 (1989), pp. 187-191.

[28] J. B. Orlin, M. A. Bonuccelli, and D. P. Bovet. An $O\left(n^{2}\right)$ algorithm for coloring proper circular-arc graphs. SIAM. J. on Algebraic and Discrete Methods 2 (1991), pp. 88-93.

[29] S.-I. Oum. Approximating rank-width and clique-width quickly. ACM Transactions on Algorithms 5 (2009), no. 1, Article 10, 20 pp.

[30] B. A. Reed. Algorithmic aspects of tree width. Recent advances in algorithms and combinatorics, pp. 85-107, CMS Books Math./Ouvrages Math. SMC, 11, Springer, New York, 2003.

[31] W. K. Shih and W. L. Hsu. An $O\left(n^{1.5}\right)$ algorithm to color proper circular arc graphs. Discrete Appl. Math. 24 (1989), no.3, pp. 321-323.

[32] R. E. Tarjan and M. Yannakakis. Simple linear-time algorithms to test chordality of graphs, test acyclicity of hypergraphs, and selectively reduce acyclic hypergraphs. SIAM J. Computing 13:3 (1984), pp. 566-579. 
[33] R. E. Tarjan. Decomposition by clique separators. Discrete Math 55 (1985), pp. 221232 .

[34] K. Vušković. Even-hole-free graphs: A survey. Appl. Anal. Discrete Math. 4 (2010), pp. $219-240$.

[35] S. H. Whitesides. A method for solving certain graph recognition and optimization problems, with applications to perfect graphs, in [3].

[36] J.R. Walter. Representations of chordal graphs as subtrees of a tree. Journal of Graph Theory 2 (1978), pp. 265-267. 